\title{
New frontiers in otorhinolaryngology and head and neck surgery
}

Volume 7 Issue I - 2017

\section{Opinion}

Today, we surgeons of otorhinolaryngology and head and neck surgery, have already started making human lifes better adaptable to the environment not only as a patient of the otorhinolaryngology and head and neck surgery but also as socially acceptable. New frontiers has been seen in almost all the aspect of the subject whether it be otology, Rhinology, laryngology, plastic surgery or facio-maxillary and dental surgery. Human lives are not only saved but also decreased from morbidity point of view.

There has been a significant shift from the age old daylight or headlight surgeries to endoscopic and high resolution special wavelength light using microscopes for the daily out-patient surgeries and major surgeries as well. Orbital decompression surgeries, endoscopic lacrimal surgeries and endoscopic management of benign endonasal tumors and malignant tumors of nose and paranasal sinuses are becoming more and more popular.

The three dimensional navigation system with reconstruction facilities and surgical techniques with the proper enhancement of the anatomy for endoscopic sinus surgeries is a boon for the surgeons of otorhinolaryngology and head and neck surgery. This has been made possible only with the new horizons in otorhinolaryngology and head and neck surgery especially the in-built 3-D imaging systems in operation theatres. Better localization of the pathological areas and disease extension have made possible to do recurrence free surgeries even in inexperienced hands.

Head and neck reconstruction surgery has gone through many changes and better esthetic compliance of the patient is seen with present form of surgeries. Mandibular reconstruction using titanium plates covered with a pedicled pectoralis major myocutaneous flap after ablative surgery for locally advanced tumors of the oral cavity along with modified radical neck dissection is a better technique for stage 4 cancer of oral cavity. It has a better esthetic effect and long term emotional compliance of the patient is good.

Narrow band imaging is becoming a boon to the early detection of laryngeal cancers. In this process special wave length light which is penetrable only to some diseased tissue is used to identify the diseased tissue. This early detection of the cancers has promised a reduction in the incidence of cancers.

Robotic surgeries are becoming more and more popular for very difficult approachable surgeries. New robotic arm surgeries for adenoidectomy, tonsillectomy and uvulopalatopharyngoplasty surgery are becoming a routine effort in day to day practice in otorhinolaryngology and head and neck surgery. Reasonable research is going on to accommodate robotic surgeries to the daily surgical procedures. The leap from the days of manual and adjustable unhealthy surgeries to this robotic world of clean cut and healthy surgeries is being looked with pessimistic attitude. Robotic drills have been used in various set-ups worldwide for otological operations like

\section{Pradip Kumar Tiwari}

Department of Otorhinolaryngology and Head and Neck Surgery Assam Medical College, India

Correspondence: Pradip Kumar Tiwari Department of Otolaryngology and Head and Neck Surgery Assam Medical College, India, Tel +9| 9864988808; +9| 9435238702, Email dr.pradiptiwari@gmail.com

Received: April 28, 2016 | Published: May 0I, 2017 cortical mastoidectomy and cochlear implant surgeries with precision and utmost care.

New instrumentations like Optical Coherence TomographyEnhanced Microlaryngoscopy, Confocal endomicrolaryngoscopy, Injection Microlaryngoscopy, Microlaryngostroboscopy, Microdebrider assisted Microlaryngoscopy, Laser Assisted Microlaryngoscopy has changed the whole scenario in otorhinolaryngology and head and neck surgery.

Optical coherence tomography is able to produce high-resolution images of vocal fold mucosa to a maximum depth of $1.6 \mathrm{~mm}$ which may be used in the diagnosis of vocal fold lesions, particularly early squamous cell carcinoma and further help in the OCT-guided microsurgery of larynx.

Confocalendomicrolaryngoscopy can detect dysplastic cells close to the basal cell layer and within the sub epithelial space in lesions with small leukoplakia. These findings will help to improve the precision for biopsy and on microlaryngoscopic laser surgery of larynx to identify the margins of the premalignant lesion.

Microlaryngostroboscopic surgery offers a very good functional result in cases of dysphonia due to a benign lesion of the vocal fold.

Microdebrider assisted Microlaryngoscopy can be an optional cyto reductive treatment and will enhance phonotary function in laryngeal diseases like angiomatous and gelatinous polypoidal lesions and papillomatosis of larynx. Laser Assisted Microlaryngoscopy for epiglottopexy in cases of laryngomalacia surgery had comparable success to other surgical techniques without having the risks of permanent scarring to the supraglottis.

Other newer instruments like Microdebrider for adenoidectomy are showing better results as recurrence is less or almost nil.

Skeeter drill for stapedectomy surgery is becoming an important tool in stapes surgery. Age old complicated procedures of stapes 
surgery can now be done within a shorter time and with greater precision. Days are not far that these procedure can be easily done by inexperienced hands.

Lasers in the treatment of otorhinolaryngology and head and neck diseases are becoming more popular and also helping in safer surgeries. Both $\mathrm{CO}_{2}$ and diode lasers are common practice in day to day surgeries. Laser helps in controlled reduction of the cancerous tissue in different modalities like thyroplasty and volume reduction laryngeal surgeries.

Ultrasonography at bedside is one of the newer frontier and a great tool in the management of otorhinolaryngology and head and neck diseases . It can easily diagnose different problems and head and neck disorders just like routine procedures in radiology department. In conjunction with the fine needle aspiration cytology it can give prompt diagnosis to different disorders and also help in early detection of head and neck cancers.

Different audiometric tests like BERA, impedence audiometry, otoacoustic emission are no more visionary but has become a part of routine investigations in the treatment of otological disorders. Cochlear implant has given a new dimension to the correction of deplorable situation specially in physically challenged, mentally retarded and differently abled patients and children. Dreams of the past are no longer dreams but has become reality.

Proper treatment also includes intervention of other disciplines like Cardio-thoracic vascular surgery, Microbiology, Pathology, Radiology, Biochemistry and Cardiology in order to avoid any following life-threatening condition. Foreign body removal from unapproachable sites are becoming easier with these interdisciplinary measures.

External beam radiation therapy represents an effective mode of treatment for patients with advanced JNA. Image-guided endoscopic coblation may be a safe and effective method for resection of intranasal portions of advance JNA tumors. The Coblator II System in many cases may limit the need for multiple instruments and improve control of intraoperative hemorrhage.

IMRT is a conformal 3-dimensional technique capable of precisely targeting tumors while avoiding normal structures and is the current standard of care in the treatment of HNSCC. IMRT refers to a controlled modulation of intensity across each individual beam so that the desired high-dose distribution matches the tumor/target in all physical dimensions. Advances in computerized treatment planning systems utilizing complex inverse planning algorithms, along with hardware improvisations including high-resolution multi-leaf collimators, have made this technique readily available for routine use at most centers in the developed world. The major benefit of IMRT compared to HRT is the sparing of critical structures while conforming the desired high doses to the tumors.

Recent advances in 3-dimensional or volumetric imaging have addressed some of these issues, and cone beam CT (CBCT) has emerged as an efficient system for in-room localization. Essentially, this consists of a compact CT scanner that is integrated into the linear accelerator unit. A scan can be acquired quickly, generally in 1 to 2 minutes, just prior to treatment with the patient in the treatment position. This scan is generally of lower resolution than a diagnostic CT scan but provides sufficient bony and soft tissue resolution for anatomic alignment. Both KV and MV CBCT systems are available, but $\mathrm{KV}$ imaging provides better image contrast and signal-noise ratio and is used at our center. The CBCT localization scan is then superimposed on the treatment planning CT scan utilizing a softwarebased registration algorithm to verify the accuracy of setup, and any necessary shifts are made to obtain an accurate match. In order to circumvent setup errors Image Guided Radiation Therapy (IGRT) that verifies the "match" between the treatment plan and final patient position and corrects for inaccuracies is currently being adapted at several centers. Treatment planning target volumes, organs at risk, and/or any user-defined regions of interest (ROI) or structures may be visualized. Bony misalignments are readily identified, rectified, and the offsets are automatically recorded. In addition, soft tissue deformations of tumor (e.g., due to regression), as well as normal structures (e.g., due to weight loss) may be identified, and it raises the possibility of "adaptive radiotherapy" (ART) in response to these changes.

Since the mid 1900s, esophagoscopy has been performed under sedation or general anesthesia. With transnasal esophagoscopy (TNE), there has been a return to awake, in-office esophagoscopy. Technologic advances have allowed the advent of a ultrathin, flexible esophagoscope that is introduced transnasally, allowing esophagoscopy to be performed in unsedated patients. TNE correlates with conventional esophagoscopy (sedated, flexible esophagoscopy) in diagnostic capacity. Over time, as the benefits of TNE have become elucidated, the procedure has gained wider acceptance and continues to have its role in patient care defined. A therapeutic application in an E.N.T office practice and also the unique technique of air insufflation with the new gadget is under study.

With the advent of chemotherapeautic drugs, cancers are no longer dreadful as it previously used to be. Single agents identified as being active in head and neck cancers include ifosfamide, methotrexate, bleomycin (Blenoxane), doxorubicin, cisplatin (Platinol), carboplatin (Paraplatin), and, more recently, docetaxel and paclitaxel (Taxol), and to a lesser extent, fluorouracil (5-FU) and the vinca alkaloids. For patients with head and neck cancers in general, combination chemotherapeutic regimens are more active than single drugs, and cisplatin-based combinations are more active than non cisplatinbased combinations. Several chemotherapeutic agents, especially cisplatin, produce synergistic and/or additive effects when used with radiotherapy. One possible advantage of using concomitant cisplatin and radiotherapy in patients with head and neck cancers, as opposed to other agents, such as methotrexate, bleomycin, or 5-FU, is the lack of increased local side effects (especially mucositis).

Several immunotherapy drugs are available and are giving a promising result without any synergistic help of surgery or chemoradiation in the treatment of diseases in otorhinolaryngology and head and neck surgery. Immunotherapy with different lactobacillus species for allergic rhinitis and sinusitis are becoming popular. One monthly course is providing an yearly satisfactory result and freedom from allergic complications. Days are not far these therapies will become a daily routine activity just like as with the gastrointestinal immunotherapy with different lactobacillus species.

Newer frontiers in endovascular therapy for different otorhinolaryngology and head and neck diseases have helped in the reduction of loss of blood during difficult surgeries like juvenile nasopharyngeal angiofibroma, sino-nasal surgeries and thyroid surgeries. Bloodless field has been of extra help to the surgeons and less time is lost for proper visualization of field.

Video and endoscopic assisted thyroid surgery is a great scaling of newer frontiers and has helped in the perfect removal of the thyroid glands with precision and scarless surgery. 
Neuro-otology and skull base surgeries have scaled to such heights that otorhinolaryngology and head and neck surgery is no more limited to small surgeries. Different and difficult surgeries for disease conditions like temporal lobe abscess and meningocoele can be easily done as routine procedures without the help of neurosurgeons. Transmastoid and retrosigmoid approach for tumors is nowadays a daily routine surgery in experienced hands and has helped the otorhino-laryngology and head and neck surgery to walk with hands with neurosurgery. This has all been possible with the day-night hardships and courage of the surgeons. Thus, it's a definite example of newer frontiers in otorhinolaryngology and head and neck surgery.

Hope and necessity keeps alive the inventions. Otorhinolaryngology and head and neck surgery is not an exception. Newer instruments like Endo-ease and ear cartilage slicers are a promising tool that will help further for the treatment of diseases. These instruments are not only a surgical tool but also an education tool for students of medical science. Better view of the operating area can be visualized and no extra help of assistants are usually required while using these instruments.

Monoclonal antibodies like Omalizumab for allergic conditions of ENT disorders, Cetuximab for head and neck cancers etc. are in evolution process for the disorders of otorhinolaryngology and head and neck diseases and their treatment. The antibodies can be used alone, or they can be used to deliver medicine or radiation directly to cancer cells to treat diseases such as leukemia and non- Hodgkin's lymphoma.
In this era of robotic technology nothing is impossible. Newer technologies for the management of traumatic injuries to the head and neck region have reached to greater heights. Today, we have the option of 3-D technology and the exact 3-dimensional xeroxed bone of the similar structure and alignment can now be printed. This new frontier is a revolution and a superb result has been seen with this technology.

These frontiers are just the beginning. We must be hopeful for betterment of every patients with present technologies. There are still more steps to be achieved and continuous efforts should be made to make life better and better.

\section{Acknowledgments}

None.

\section{Conflicts of interest}

Author declares there are no conflicts of interest.

\section{Funding}

None. 